\title{
In memoriam Professor Dr. med. Maschallah Nadjmi †
}

\begin{abstract}
A m 13. Juni 2000 ist Professor Dr. med. Maschallah Nadjmi, emeritierter Leiter der Abteilung für Neuroradiologie in der Kopfklinik der Universität Würzburg, im Alter von 71 Jahren plötzlich verstorben. Mit ungläubiger Bestürzung haben wir diese Nachricht aufgenommen, hatte er doch seinen Ruhestand in solch beneidenswerter geistiger und körperlicher Frische und voller sportlicher Aktivitäten genossen, dass jeder Gedanke an Krankheit oder gar Tod abwegig erschien.
\end{abstract}

Am 25. Mai 1929 wurde er in Teheran geboren. Dort besuchte er die Volksschule und anschließend die Oberrealschule Alborz (American College), wo er 1948 die Reifeprüfung bestand. Im Jahre 1949 begann er mit seinem Medizinstudium in Teheran und setzte es ab 1950 in Hamburg, später in Freiburg fort. 1954 bestand er in Freiburg das Ärztliche Staatsexamen. Im Jahre 1959 promovierte er in Köln bei Tönnis über den „Histochemischen Nachweis von Vitamin $\mathrm{C}$ in den Hypophysenadenomen“ mit der Note „magna cum laude“. Die Gutachter waren Tönnis und Zülch.

Seine ärztliche Tätigkeit nahm er 1955 unter Tönnis in der Neurochirurgischen Klinik der Universität Köln auf. Nach anderthalb Jahren wechselte er in die Medizinische Abteilung des St.-Elisabeth-Krankenhauses in Köln. Seit dem 1. September 1957 war er am Universitätsklinikum in Würzburg in der Neurologischen Klinik unter Schaltenbrand tätig. Er erhielt 1971 die Facharztanerkennung für Nervenheilkunde und 1983 für Radiologie.

Seine wissenschaftlichen Aktivitäten auf dem Gebiet der Neuroradiologie und Stereotaxie führten 1965 zur Habilitation über „Die Radiologie der diencephalen hypophysären Region als Grundlage für die Stereotaxie“. 1971 wurde er zum außerplanmäßigen Professor, 1972 zum Leiter der Neuroradiologie ernannt. Diese erhielt - nicht zuletzt durch sein großes Engagement im Jahre 1977 den Status einer selbständigen Abtei-

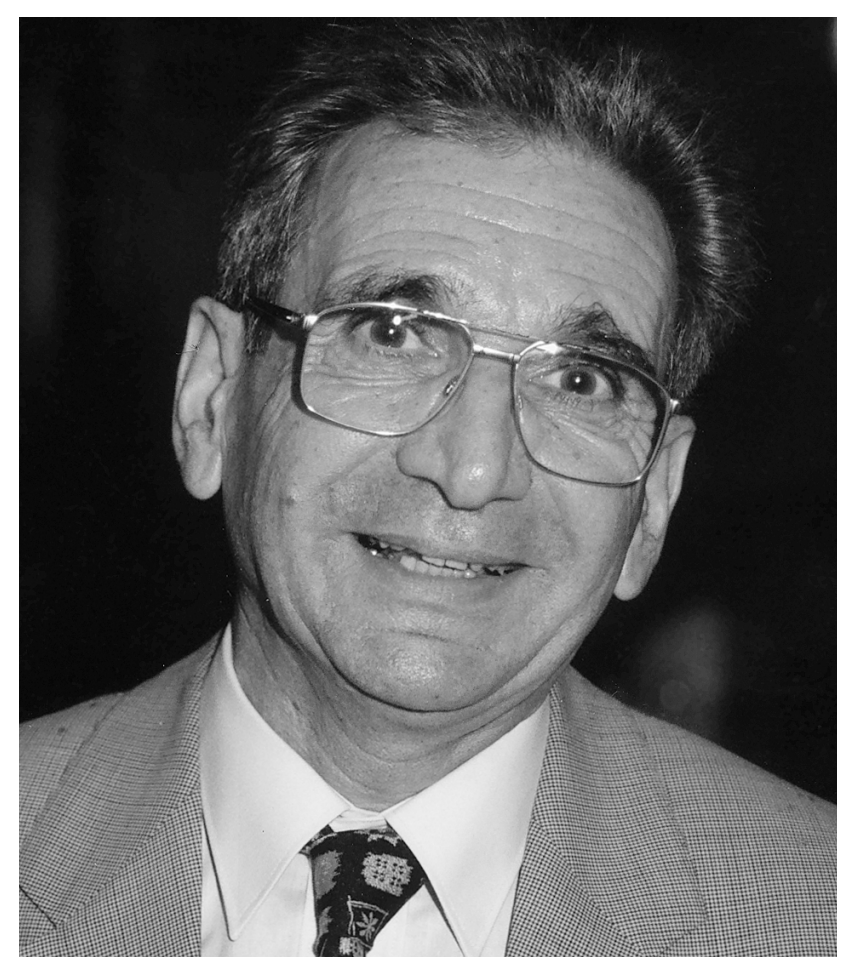

lung der Universität Würzburg; er wurde zum Universitätsprofessor für das Fach Neuroradiologie berufen.

Den Höhepunkt seiner wissenschaftlichen Aktivitäten markiert der Anbruch des digitalen Zeitalters in der Neuroradiologie. So entwickelte Nadjmi noch Mitte der siebziger Jahre die Tomosynthese als letztes analoges Schnittbildverfahren, erkannte aber früh das Entwicklungspotenzial der Computertomographie und machte Würzburg zu einem der ersten deutschen CT-Standorte. Er konnte als federführender Herausgeber eines der ersten CT-Atlanten der Neuroradiologie, der heute ein Klassiker ist, einen weiteren Meilenstein setzen. Auch die frühen achtziger Jahre waren geprägt von hoher Innovationsfreude: Die Einführung der digitalen Subtraktionsangiographie schlug sich in einem weiteren Lehrbuch nieder, und schließlich war es wieder Würzburg, wo einer der ersten hochauflösenden Computer- 
tomographen in Deutschland aufgestellt wurde. In der letzten Phase seines Berufslebens widmete sich Nadjmi vor allem der interventionellen Neuroradiologie. Es war nicht zu übersehen, dass er sie als besondere persönliche Herausforderung auffasste, auch wenn sie ihm einen hohen physischen Einsatz abverlangte.

Den Studenten präsentierte er die Neuroradiologie als dynamisches und spannendes klinisches Fach. Seine Kurse und Vorlesungen wurden daher ausgesprochen gerne besucht. Dies schlug sich regelmäßig in Bestnoten nieder, wenn die Studenten die ihnen angebotenen Lehrveranstaltungen intern bewerteten.

Er war Gründungsmitglied und einer der ersten Präsidenten der Deutschen Gesellschaft für Neuroradiologie (1973 bis 1974). Nadjmi war Kosmopolit und dennoch die Triebfeder für eine selbstbewusste Integration aller Deutsch sprechenden Neuroradiologen. Diesem Ziel konnte gerade er sich unvorbelastet und unvoreingenommen stellen. Einer der Erfolge dieses Strebens ist diese Zeitschrift, die er mit großem Idealismus zum Organ der neuroradiologischen Fachgesellschaften Österreichs, der Schweiz und Deutschlands ausbaute.

Nadjmi holte zweimal die Jahrestagung der Deutschen Gesellschaft für Neuroradiologie nach Würzburg. Unvergesslich ist aber vor allem der Kongress der Euro- pean Society of Neuroradiology im Jahre 1988, den er gemeinsam mit seiner Frau Christa als herzlicher und charmanter Gastgeber prägte.

Nadjmi war bei oberflächlicher Betrachtung ein überaus liberaler Mensch. Man spürte aber immer wieder die innere Spannung zwischen seiner Herkunft aus dem Orient und seinem langjährigen Leben und Wirken im Okzident. Einen Teil seiner produktiven Unruhe hat er aus diesem Spannungsverhältnis bezogen. Er war in seiner Abteilung die uneingeschränkte Autorität, drängte sich aber nie auf und schuf damit eine wichtige Voraussetzung für seine Mitarbeiter, sich an ihrem Arbeitsplatz gut aufgehoben zu fühlen.

Nadjmi hat wesentlich zum Ansehen der Neuroradiologie beigetragen. Er war dennoch von beispielhafter Bescheidenheit, wenn es um seine eigene Person ging. Er hasste Personenkult. Berufliche oder private Jubiläen und Zäsuren waren ihm suspekt und keinesfalls Anlass für irgendwelche Feierlichkeiten. Daher haben seine Angehörigen und Freunde in aller Stille von ihm Abschied genommen. Hiermit verabschieden sich von ihm seine Schüler und Mitstreiter. Die Deutsche Gesellschaft für Neuroradiologie trauert um eines der namhaftesten Mitglieder ihrer Gründerzeit.

Erich Hofmann

László Solymosi 